\title{
Circulating MicroRNAs predict glycemic improvement and response to a behavioral intervention
}

\author{
Elena Flowers ${ }^{1,2^{*}}$ (D), Isabel Elaine Allen ${ }^{3}$, Alka M. Kanaya ${ }^{3,4}$ (D) and Bradley E. Aouizerat ${ }^{5,6}$ (D)
}

\begin{abstract}
Background: MicroRNAs may be important regulators of risk for type 2 diabetes. The purpose of this longitudinal observational study was to assess whether circulating microRNAs predicted improvements in fasting blood glucose, a major risk factor for type 2 diabetes, over 12 months.

Methods: The study included participants $(n=82)$ from a previously completed trial that tested the effect of restorative yoga on individuals with prediabetes. Circulating microRNAs were measured using a flow cytometry miRNA assay. Linear models were used to determine the optimal sets of microRNA predictors overall and by intervention group.
\end{abstract}

Results: Subsets of microRNAs were significant predictors of final fasting blood glucose after 12-months $\left(R^{2}=0.754\right.$, $p<0.001)$ and changes in fasting blood glucose over 12 -months $\left(R^{2}=0.731, p<0.001\right)$. Three microRNAs (let-7c, miR-363, miR-374b) were significant for the control group only, however there was no significant interaction by intervention group.

Conclusions: Circulating microRNAs are significant predictors of fasting blood glucose in individuals with prediabetes. Among the identified microRNAs, several have previously been associated with risk for type 2 diabetes. This is one of the first studies to use a longitudinal design to assess whether microRNAs predict changes in fasting blood glucose over time. Further exploration of the function of the microRNAs included in these models may provide new insights about the complex etiology of type 2 diabetes and responses to behavioral risk reduction interventions.

Trial registration: This study was a secondary analysis of a previously completed clinical trial that is registered at clinicaltrials.gov (NCT01024816) on December 3, 2009.

Keywords: microRNA, Diabetes, Fasting blood glucose, Biomarker, Yoga

\section{Background}

Type 2 diabetes (T2DM) affects more than 460 million individuals globally [1] and is associated with $\$ 245$ billion in costs annually in the United States alone. [2]

\footnotetext{
* Correspondence: elena.flowers@ucsf.edu

'Department of Physiological Nursing, University of California, San Francisco, 2 Koret Way, \#605L, CA 94143-0610 San Francisco, USA

${ }^{2}$ Institute for Human Genetics, University of California, San Francisco, 2 Koret Way, \#605L, CA 94143-0610 San Francisco, USA

Full list of author information is available at the end of the article
}

Individuals with T2DM are at risk for a number of serious complications, including cardiovascular diseases, retinopathy, and renal disease. [2] One of the primary challenges to preventing and treating T2DM is the incomplete understanding of its multifactorial etiology. [3] While a number of genetic risk factors for T2DM that provide some insight into the mechanisms underlying T2DM have been reported [4], modifiable lifestyle and behavioral characteristics are equally important risk

C C The Author(s). 2021 Open Access This article is licensed under a Creative Commons Attribution 4.0 International License, which permits use, sharing, adaptation, distribution and reproduction in any medium or format, as long as you give appropriate credit to the original author(s) and the source, provide a link to the Creative Commons licence, and indicate if changes were made. The images or other third party material in this article are included in the article's Creative Commons licence, unless indicated otherwise in a credit line to the material. If material is not included in the article's Creative Commons licence and your intended use is not permitted by statutory regulation or exceeds the permitted use, you will need to obtain permission directly from the copyright holder. To view a copy of this licence, visit http://creativecommons.org/licenses/by/4.0/. The Creative Commons Public Domain Dedication waiver (http://creativecommons.org/publicdomain/zero/1.0/) applies to the data made available in this article, unless otherwise stated in a credit line to the data. 
factors. The growing appreciation for the large impact of this latter category of risk factors on genetic risk, broadly characterized as gene-environment interactions, has resulted in a focus on identifying and characterizing biomarkers that can reflect these complex relationships. A clearer understanding of gene-environment interactions that influence risk for T2DM may allow for better specification of risk-reduction interventions for T2DM or facilitate development of new interventions based on improved understanding of the underlying interactions.

Development of T2DM occurs on a continuum spanning normal blood glucose to prediabetes to T2DM. Fasting blood glucose (FBG) is the biomarker used to assess for risk for T2DM [5] and is easily measured in both clinical settings and a patient's home environment. FBG can characterize glycemic variability over time, glycemic progression (e.g., from normal glucose tolerance to prediabetes or from prediabetes to T2DM), as well as glycemic improvement in response to interventions. Even prior to a T2DM diagnosis, T2DM related complications can begin to develop, making FBG an important tool for monitoring which individuals are in greatest need of interventions. The possibility for novel prodromal biomarkers that capture harmful physiological changes prior increased FBG could further improve detection of risk and prevention of T2D and related complications.

MicroRNAs (miRs) are short (i.e., 18-26 nucleotide) regulatory elements of messenger RNA translation to amino acids. Because miRs regulate gene expression, they operate as a function of both underlying genetic risk for disease as well as environmental factors such as responses to risk reduction interventions, including behavioral factors. [6, 7] Circulating miRs are easily measured from blood serum or plasma and are potential biomarkers for risk for development of T2DM, [8-10] exhibiting changes in expression levels prior to the onset of T2DM. [11, 12] There are at least two potential applications of miRs as predictive biomarkers related to T2DM: the first is improved identification of which individuals are at greatest risk for progression towards T2DM; and the second is identification of individuals who are likely to respond to risk reduction interventions, including behavioral interventions. Because miRs capture changes in physiology before the onset of elevated FBG, they have the potential to be important prodromal markers of risk for T2D.

Our prior clinical trial (i.e., Practicing Restorative Yoga vs. Stretching for the Metabolic Syndrome (PRYSMS); clinicaltrials.gov identifier NCT01024816) showed that a restorative yoga intervention was effective at decreasing FBG compared to stretching in individuals with the metabolic syndrome. [13] One of the hypothesized mechanisms underlying the beneficial effect of restorative yoga was decreased level of stress. However, a secondary analysis of the PRYSMS trial showed that cortisol levels and self-reported measures of stress decreased more in participants from the stretching control group than in the yoga intervention group. [14] Therefore, the mechanisms underlying the beneficial effects of restorative yoga on FBG remain largely unknown. Circulating miRs, by characterizing effects of behavioral interventions on underlying genetic predisposition, may be useful biomarkers of responses to and may provide new insights about the underlying mechanisms of restorative yoga.

The goal of this study was to characterize the associations between miRs and FBG both after 12-months as well as change in FBG from baseline to 12-months. To our knowledge, this is the first study to leverage a longitudinal design to determine whether miRs might be useful biomarkers to identify which individuals are likely to respond to risk reduction interventions for T2DM and which miRs might correspond with changes in FBG and therefore be potential future mechanistic targets.

\section{Methods \\ Participants}

The study sample included participants from the previously completed Practicing Restorative Yoga versus Stretching for the Metabolic Syndrome (PRYSMS) clinical trial (clinicaltrials.gov identifier NCT01024816), which tested the effects of restorative yoga versus active stretching on FBG in overweight adults at risk for T2DM. Participants in the PRYSMS study were recruited from the San Francisco and San Diego areas and met the International Diabetes Federation criteria for metabolic syndrome. [15]. Exclusion criteria from the PRYS MS trial included FBG $\geq 126 \mathrm{mg} / \mathrm{dl}$, hemoglobin A1c $(\mathrm{HbA} 1 \mathrm{c}) \geq 7.0 \%$, fasting triglycerides $\geq 300 \mathrm{mg} / \mathrm{dl}$, weight $\geq 400$ pounds, neurological conditions that limited mobility, hospitalization for coronary heart disease within the past 6 months, current pregnancy or lactation, history of bariatric surgery, substance abuse, and use of medications affecting metabolic factors. Demographic and behavioral characteristics and medical history were collected by trained study personnel at the baseline visit.

\section{Study Design}

Randomization was stratified by sex and race/ethnicity and participants were assigned to either the restorative yoga intervention or the active stretching group. Both interventions were delivered in a group setting twice weekly for the first 12-weeks, then weekly for 12-weeks, then monthly for the remainder of the trial. In addition, all participants received a presentation on healthy behaviors, including nutrition and physical activity information. 


\section{Clinical Data Collection}

Clinical data were collected at baseline, 3-, 6-, 9-, and 12-months. Participant weight was measured on a standard balance beam scale and height using a stadiometer. Waist circumference was measured using a Gullick II tape spring-tension measure at the site of maximum circumference midway between the lower ribs and the anterior superior iliac spine. The mean of two waist circumference measurements was calculated. Body mass index (BMI) was calculated as weight in kilograms divided by height in meters squared.

FBG was measured using an automated analyzer with an immobilized enzyme biosensor (YSI 2300 STAT Plus, YSI Life Sciences, Yellow Sprints, OH). Total cholesterol, triglycerides and HDL-cholesterol were measured by enzymatic calorimetric methods (Quest Diagnostics, San Jose, CA), and LDL-cholesterol was calculated using the Friedewald equation [16]. Blood used for banking of plasma was collected by venipuncture into vacutainers containing the preservative EDTA, centrifuged at $4{ }^{\circ} \mathrm{C}$ to separate plasma from cellular blood components, and stored at $-80{ }^{\circ} \mathrm{C}$.

\section{Molecular Data Collection}

Plasma specimens were banked at the same time points as when clinical data were obtained (i.e., baseline, 3months, 6-months, 9-months, and 12-months) for the parent trial. The Firefly Bioworks Multiplex Circulating MicroRNA Assay (Abcam, MA) was used for direct quantification of miRs from plasma at all five timepoints. MiRs were hybridized to complementary oligonucleotides covalently attached to encoded hydrogel microparticles. The bound target was ligated to oligonucleotide adapter sequences that serve as universal PCR priming sites. The miR-adapter hybrid models were then denatured from the particles and reverse transcription polymerase chain reaction (RT-PCR) was performed using a fluorescent forward primer. Once amplified, the fluorescent target was rehybridized to the original capture particles and scanned on an EMD Millipore Guava 6HT flow cytometer (Merck KGaA Darmstadt, Germany). Expression levels of 59 miRs (Supplemental Table 1) were measured from plasma specimens collected at each of the five timepoints on a subset of 86 participants from the PRYSMS trial. The selection of these 59 miRs was based on a previous discovery analysis from a larger set of miRs in an independent subset of participants from the PRYSMS trial (manuscript in press). In this discovery analysis, expression levels of 336 reliably detectable circulating miRs were measured on a subset of 10 participants in 2016 and 402 reliably detectable circulating miRs were measured from an additional subset of 10 participants in 2018. The 59 samples included in the study described in this manuscript represent the union of all miRs that were significantly differentially expressed between individuals with normal and stable FBG levels compared to those with elevated and highly variable FBG over the 12-month trial period in the discovery analyses. All miRs and all sample wells included in this experiment passed quality control measures, which included a signal $>1,000$ arbitrary units or detection of a spike-in control and a blank signal from particles with no microRNA probes.

The study described here included the subset of participants with banked plasma specimens from the baseline study visit with availability of at least two additional follow-up timepoints $(n=82)$. Samples were not previously thawed.

\section{Statistical Analysis}

Descriptive statistics were calculated to examine and evaluate the demographic and clinical characteristics of participants. (R, 2019) Means and standard deviations are reported for continuous variables and counts and percentages are reported for categorical variables. Comparisons between the intervention and control groups used independent groups Student's t-test for continuous variables and chi-squared tests for categorical variables. Missing values for FBG were imputed using the mean FBG for the individual participant.

The absolute number and range of copies of individual miR that occur in a biological sample, as estimated using the flow cytometry-based Firefly assay in arbitrary units (AU), can vary considerably. To control for the differences in the scale of the miRs measured by AUs, miRs were standardized using z-scores to facilitate cross-miR comparisons of estimates of association with FBG measures.

A limitation of approaches to adjustment for multiple comparisons is that they may not be appropriate given the underlying assumption that all tests are independent, whereas the roles of individual miRs associated with a given physiological process are likely not independent. In this study, we applied a data-driven approach to determine which combination of miRs (of all possible combinations of the 59 measured miRs) accounted for the largest estimated proportion of the variability (i.e., $\mathrm{R}^{2}$ value) for both FBG after 12-months and change in FBG from baseline to 12 -months. MiRs were used in linear models alone and then with adjustment for statistically relevant covariates. Models were fit overall and by intervention group. For the analyses stratified by group, $p<$ 0.10 was used as a criterion for selection in order to identify miR predictors that might otherwise be missed because of multicollinearity. [17] Estimates of association and statistical test of significance for each covariate and the overall models are reported. All statistical modeling was done using Stata version 16.1, College Station, TX. 
Expression of individual miRs was normalized using the set of miR probes (i.e., hsa-miR-92a-3p, hsa-mir-93$5 \mathrm{p}$, hsa-miR-17-5p) identified by the geNorm algorithm for each experiment. [18] All included miR targets passed quality control measures and were retained in the analysis.

For the miRs that were included in the models, box and whisker plots were created in order to assess the overall variability in individual miRs (Microsoft Excel 2019, Redmond WA).

The TargetScan database was used to identify predicted messenger RNA (mRNA) targets of the miRs that were identified as optimal predictors of FBG and identify which miRs are within families. [19]

\section{Results}

\section{Participant Characteristics}

A total of 82 participants from the PRYSMS trial were included in this analysis. The mean age was $55 \pm 7$ years, the majority identified as female (73\%) and White (70\%) and had a college degree or higher level of education (66\%). The study sample was obese with a mean BMI of $35.1 \pm 7.2 \mathrm{~kg} / \mathrm{m}^{2}$. Overall, the group met the criteria for prediabetes with a mean FBG of $104 \pm 13 \mathrm{mg} /$ dL. Additional demographic and clinical characteristics in the overall sample and by intervention group are shown in Table 1 and Supplemental Tables 2 and 3. In this subset of 82 participants from the PRYSMS trial, FBG at baseline was $105 \pm 13 \mathrm{mg} / \mathrm{dL}$ in the intervention group compared to $100 \pm 11 \mathrm{mg} / \mathrm{dL}$ in the control group $(p=0.08)$. The change in FBG over 12-months was $4 \pm 10 \mathrm{mg} / \mathrm{dL}$ in the intervention group compared to $9+$ $40 \mathrm{mg} / \mathrm{dL}$ the control group $(p<0.05)$.

\section{Associations Between miRs and FBG}

Of the 59 measured miRs, none were independently associated with FBG after 12-months or the change in FBG after 12-months in univariate models.

Using a general linear model, $14 \mathrm{miRs}$ (let-7c, miR-17, miR-20b, miR-22, miR-92a, miR-93, miR-106b, miR167d, miR-192, miR-197, miR-296, miR-342, miR-363, miR-374b) were identified as the optimal set of miR predictors of FBG at 12-months with an overall model Rsquared of $0.754(p<0.001)$ (Table 2). Addition of the three covariates that differed between the intervention and control group (i.e., waist and hip circumference, weight) did not change the observed differences, nor were these variables significant in the overall model. For change in FBG after 12-months, 12 miRs (let-7c, miR17, miR-20b, miR-22, miR-92a, miR-93, miR-106b, miR186, miR-192, miR-296, miR-342, miR-374b) were identified as the optimal set of predictors with an overall model R-squared of $0.731 \quad(p<0.001)$ (Table 2). To
Table. 1 Demographic and Clinical Characteristics of the Sample

\begin{tabular}{ll}
\hline $\mathbf{n}$ (\%) oraverage \pm standard deviation & $\mathbf{n = 8 2}$ \\
\hline Age (years) & $55 \pm 7$ \\
Male sex (n) & $22(27)$ \\
Completed College & $54(66)$ \\
Race & \\
Asian & $11(13)$ \\
Black & $4(5)$ \\
Latin & $8(10)$ \\
White & $57(70)$ \\
Other/Mixed & $2(2)$ \\
Glucose (serum) (mg/dL) & $104 \pm 13$ \\
Total Cholesterol (mg/dL) & $206 \pm 39$ \\
Triglycerides (mg/dL) & $167 \pm 63$ \\
LDL Cholesterol (mg/dL) & $125 \pm 35$ \\
HDL Cholesterol (mg/dL) & $49 \pm 11$ \\
Body Mass Index (kg/m ${ }^{2}$ ) & $35.1 \pm 7.2$ \\
Waist Circumference (cm) & $110 \pm 13$ \\
Hip Circumference (cm) & $117 \pm 12$ \\
Weight (pounds) & $211 \pm 43$ \\
Systolic Blood Pressure (mmHg) & $124 \pm 15$ \\
Diastolic Blood Pressure (mmHg) & $72 \pm 8$ \\
\hline
\end{tabular}

$\mathrm{cm}$ centimeters; $d L$ deciliters; $H D L$ high density lipoprotein; $\mathrm{kg}$ kilograms;

$L D L$ low density lipoprotein; $m$ meters; $m g$ milligrams; $m m H g$ millimeters

of mercury

facilitate interpretation of single unit changes in miR expression (in AU) to single unit change in FBG (mg/dL), non-standardized values for individual miRs that were significant predictors in the models are shown in Table 3.

There was no statistically significant interaction with intervention group for FBG at 12-months or change in FBG over 12 months. However, in stratified analyses by intervention group, there were some differences in the subset of miRs identified as the optimal predictors of FBG. The active control group retained five miRs that were independently statistically significant (i.e., let-7c, miR-92a, miR-93, miR-363, miR-374b), whereas the intervention group retained only two miRs (miR-92a, miR-93) with independent statistical significance $(p<0.1$ for all). These associations were the same for both FBG outcomes (i.e., at 12 months, change from baseline at 12 months) (Supplemental Tables 4 and 5).

The full list of all predicted mRNA targets of miRs identified in the models is shown in Supplemental Table 6. There are a total of 11,584 predicted miRmRNA pairings. Some of the identified miRs in both predictive models are within families. There two sets of families identified. The first includes miR-17-5p, miR- 
Table 2 Standardized ${ }^{\mathrm{a}}$ MicroRNA Predictors of Fasting Blood Glucose

\begin{tabular}{|c|c|c|c|c|c|c|}
\hline \multirow{3}{*}{$R^{2}(p$-value $)$} & \multicolumn{3}{|c|}{ Fasting Blood Glucoseat 12-Months } & \multicolumn{3}{|c|}{ Change in Fasting Blood Glucose after 12-Months } \\
\hline & \multicolumn{3}{|c|}{$0.754(p<0.001)$} & \multicolumn{3}{|c|}{$0.731(p<0.001)$} \\
\hline & $\beta$ & SE & $p$-value & $\beta$ & SE & $p$-value \\
\hline (Constant) & 116.835 & 9.455 & $<0.001$ & & & \\
\hline let-7c-5p & 57.793 & 17.213 & 0.006 & 36.473 & 15.538 & 0.039 \\
\hline $\operatorname{miR}-17-5 p$ & 103.369 & 56.573 & 0.095 & 115.762 & 51.070 & 0.045 \\
\hline miR-20b-5p & -44.197 & 19.784 & 0.047 & -46.590 & 17.859 & 0.024 \\
\hline $\operatorname{miR}-22-3 p$ & 54.453 & 21.730 & 0.029 & 40.900 & 19.616 & 0.061 \\
\hline miR-92a-3p & 153.475 & 75.753 & 0.068 & 155.501 & 68.383 & 0.044 \\
\hline miR-93-5p & 109.430 & 47.467 & 0.042 & 102.078 & 42.849 & 0.036 \\
\hline$m i R-106 b-5 p$ & -36.680 & 12.263 & 0.012 & -26.795 & 11.070 & 0.034 \\
\hline mir-186-5p & -10.961 & 22.123 & 0.283 & -12.247 & 23.123 & 0.456 \\
\hline miR-192-5p & -57.402 & 21.394 & 0.021 & -41.995 & 19.312 & 0.052 \\
\hline miR-197-3p & 82.176 & 32.636 & 0.029 & & & \\
\hline miR-296-5p & 42.881 & 13.913 & 0.010 & 35.448 & 12.560 & 0.017 \\
\hline miR-342-3p & 29.558 & 11.615 & 0.027 & 25.671 & 10.485 & 0.032 \\
\hline miR-363-3p & 24.191 & 11.045 & 0.051 & & & \\
\hline miR-374b-5p & 40.072 & 22.111 & 0.097 & 36.522 & 19.960 & 0.094 \\
\hline
\end{tabular}

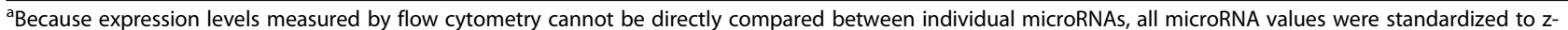
scores so that the mean expression level is equal to zero and a 1-unit change is equal to one standard deviation from the mean

$\beta$ beta value; SE standard error

20b-5p, miR-93-5p, and miR-106-5p. The second includes miR-92a-3p and miR-363-3p.

\section{Discussion}

This study assessed whether microRNAs were significant predictors of FBG after a 12-month behavioral intervention in participants with prediabetes. Using linear modelling, we assessed both FBG at 12 months and the change in FBG after 12 months and identified 14 and 12 miRs that were statistically significant predictors, respectively. The models overall accounted for significant variability in both FBG outcomes (i.e., $\mathrm{R}^{2}>0.7$ ). While no evidence of statistical interaction by intervention group was observed, three miRs were significant predictors of FBG in the active control group but not the intervention group, suggesting the effects of the intervention on FBG may be mediated by miRs. Finally, we assessed the variability of the significant miR predictors from the model for FBG after 12 months and identified miRs with the greatest variability and potential for a greater physiological range.

Prior studies have typically focused on the associations of single miRs with T2DM and related outcomes based on a priori hypotheses or agnostic hypothesis-generating

Table 3 Non-standardized microRNA values associated with fasting blood glucose outcomes

\begin{tabular}{|c|c|c|c|c|c|c|}
\hline \multirow{3}{*}{$R^{2}(p$-value $)$} & \multicolumn{3}{|c|}{ Final Fasting Blood Glucoseat 12-Months } & \multicolumn{3}{|c|}{ Change in Fasting Blood Glucose after 12-Months } \\
\hline & \multicolumn{3}{|c|}{$0.873(p<0.001)$} & \multicolumn{3}{|c|}{$0.734(p<0.001)$} \\
\hline & $\beta$ & SE & $p$-value & $\bar{\beta}$ & SE & $p$-value \\
\hline miR-106b-5p & -0.007 & 0.009 & 0.431 & -0.010 & 0.013 & 0.431 \\
\hline let7c-5p & -0.003 & 0.009 & 0.763 & -0.007 & 0.015 & 0.659 \\
\hline miR-20b-5p & -0.003 & 0.004 & 0.529 & 0.003 & 0.006 & 0.664 \\
\hline miR-296-5p & -0.184 & 0.091 & 0.045 & -0.237 & 0.150 & 0.120 \\
\hline miR-342-3p & 0.020 & 0.017 & 0.257 & 0.068 & 0.028 & 0.019 \\
\hline miR-92a-3p & 0.023 & 0.003 & 0.000 & 0.022 & 0.003 & 0.000 \\
\hline mir-93-5p & 0.021 & 0.005 & 0.000 & 0.021 & 0.004 & 0.000 \\
\hline miR-17-5p & 0.016 & 0.004 & 0.000 & 0.014 & 0.003 & 0.000 \\
\hline
\end{tabular}

$\beta$-values represent single unit change in flow cytometry-measured fluorescence signal (arbitrary units (AU)) that corresponds to a single unit change in FBG $(\mathrm{mg} / \mathrm{dL})$

$\beta$ beta; FBG fasting blood glucose; SE standard error 
approaches with correction for multiple comparisons. In contrast, we sought to use linear modelling to derive the set of miRs that was the most accurate for prediction of FBG outcomes. There is a growing consensus that miRs act synergistically through co-regulation of individual genes and sets of genes that have related functions or are co-located in biological pathways. [20] A limitation of the agnostic approach with adjustment for multiple comparisons is that it may not be appropriate given the underlying assumption that all tests are independent, whereas the roles of individual miRs associated with a given physiological process are likely not independent. In this study, we applied a data-driven approach to determine which combination of miRs accounted of the largest estimated proportion of the variability for both FBG after 12-months and change in FBG from baseline to 12-months. This approach not only allows for greater statistical power by including a set of miR predictors, but also offers the possibility of identifying sets of miRs that may co-regulate genes, sets of genes, and biological pathways that underlie risk for T2DM. For example, both miR-192 and miR-20b target components of peroxisome proliferator activated receptor gamma (PPARG) [21], which is located in the Type II diabetes mellitus pathway [22]. PPARG is primarily expressed in adipose tissue, has a role in regulation of adipocyte differentiation, and has been associated with risk for obesity and T2DM. [23] In addition, this receptor is the target of the thiazolidenidone class of medications that are used to improve insulin sensitivity and decrease risk for T2DM or control FBG in individuals diagnosed with T2DM. In silico analysis of the predicted mRNA and biological pathway targets of the sets of miRs associated with FBG may provide additional information about larger networks that underlie risk for T2DM. Further, the miRs identified by these models are potential therapeutic targets that may be able to impact multiple mechanisms underlying risk for T2DM.

For most miRs, the complete biological implications are not fully known. This study validated a subset of the findings observed in our preliminary discovery analysis of an independent subgroup of participants from the PRYSMS study sample. For example, in an independent sample, miR-192, miR-93, and miR-197 were previously found to be associated with variability (i.e., individuals showing high variability in FBG compared to individuals with stable FBG) over 12-months. We also previously observed cross-sectional associations between miR-106b and miR-22 and baseline FBG. [24] In another study sample, we observed associations between miR-197 and glycemic progression over 12-months [12] and let-7c and 106b showed changes in expression following a 3month behavioral intervention for weight loss [24]. There is evidence that miR-93 has a role in processes related to T2DM, including insulin resistance and subsequent polycystic ovarian syndrome [25], and a prior genome-wide association study found an association between insulin like growth factor binding protein 5 (IGFBP5), which is a mRNA target of miR-197, and adipose tissue volume in women. [26] Our own recent in silico analyses of the $57 \mathrm{miRs}$ evaluated here showed clear enrichment of target genes on pathways relevant to T2DM. For example, AKT1 is a mRNA target of miR192. AKT1 is highly expressed in the pancreas and liver, both of which are related to the pathophysiology of T2DM, including skeletal muscle uptake of glucose, glucose metabolism and lipogenesis in adipose tissue, and hepatic glucose production. [27] Multiple studies showed decreased expression of miR-106b following bariatric surgery. [28] MiR-106b is associated with GLUT4 expression and translocation in response to insulininduced glucose uptake in skeletal muscle. [29] In vitro studies of human adipocyte differentiation showed consistent changes in expression of miR-106b in mature adipocytes compared to their precursors, and miR-106b targets genes located in pathways associated with adipocyte differentiation. [30]

For the remaining miRs associated with FBG, the biological functions are less clear, but associations with T2DM and related conditions have been reported. MiR let-7c is as a potential therapeutic target to mediate the activity of transforming growth factor $\beta 1$ (TGF- $\beta 1)$ in diabetic kidney disease and [31] and polarization of macrophages in immune responses [32]. MiR-186, [33] miR342, [34] miR-363, [35] miR-374, [36] and miR-92a [37] have been identified in studies of vascular endothelial cell function and therefore may be more related to complications from, rather than the etiology of, T2DM. Similarly, miR-296 appears to target sodium-glucose transporter-2 (SGLT2) to promote wound healing in individuals with T2DM [38]. Additional studies exploring the functional role of these miRs related to T2DM and whether they can serve as predictive markers of not only T2DM but also related complications are needed.

MiRs with narrow physiological ranges (i.e., low variability in expression levels) may be difficult to manipulate for therapeutic purposes and result in off-target effects. Conversely, manipulation of miRs that show a greater dynamic range may be better tolerated and more variable miRs may be better therapeutic targets. Therapeutic manipulation of miRs can occur through administration of synthetic miR mimics or inhibitors that bind to endogenous miRs and prevent binding to their mRNA targets. The variances of the miRs included in models for FBG after 12-months are shown in Fig. 1. Let-7c, miR-20b, and miR-22 show relatively greater variance compared to others. Preliminary evidence supports associations between miR-20b and risk for T2DM, including 

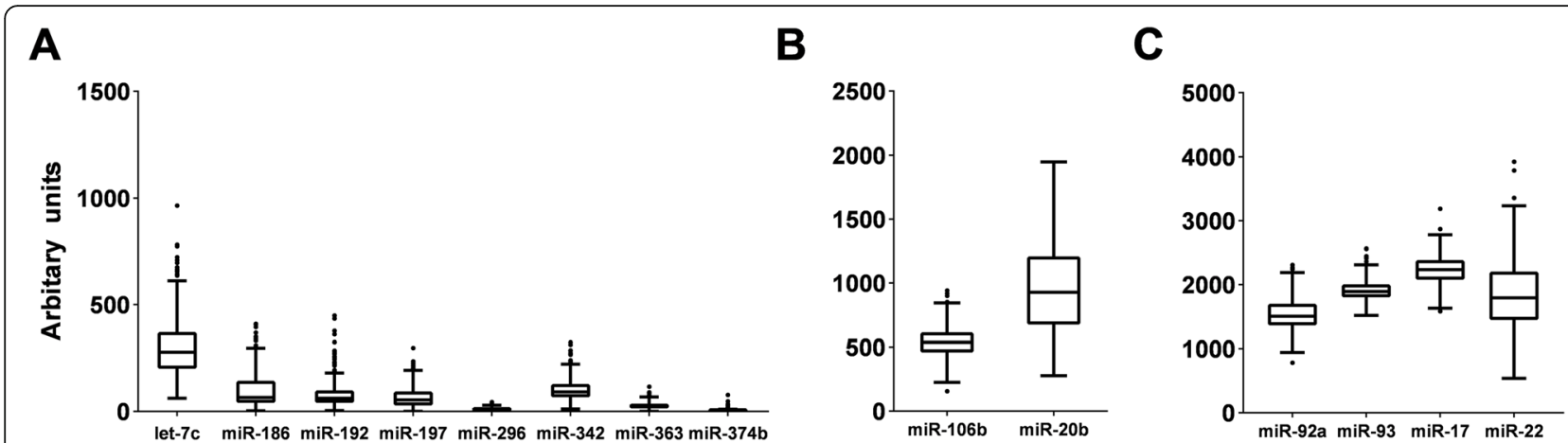

Fig. 1 Box and Whisker Plots Depicting Variability in MicroRNA Expression Levels. Within each box, the bottom border represents the 25th percentile, the center line represents the 50th percentile, and the upper border represents the 75 th percentile. The lowest horizontal line represents the minimum, and the upper horizontal line represents the maximum. Small black circles represent outliers. Individual microRNAs are represented on the $x$-axis. Arbitrary florescence units (AU) are represented on the $y$-axis. Because the overall range of AU was large, subsets of microRNAs were grouped into panels. Panel A shows microRNAs with median AUs < 500. Panel B shows microRNAs with AUs between 500-1000, Panel C shows microRNAs with AUs $>1000$

insulin uptake in skeletal muscle [39] and T2DM-related complications including diabetic retinopathy [40] and endothelial cell function [41]. Similarly, miR-22 has been associated with adipose tissue mass, insulin sensitivity, and glucose homeostasis [42] and diabetic cardiomyopathy [43]. Evaluation of which miRs have a high relative variance and show evidence for associations with risk for T2DM may prioritize which miRs may be therapeutic targets controlling or decreasing FBG and preventing onset of T2DM.

Three microRNAs (i.e., let-7c, miR-363, miR-374b) were significant contributors to the models for both final FBG and change in FBG in the active control group but not the restorative yoga intervention group (Table 4). This finding may provide some insights into the potential mechanisms underlying the effect of restorative yoga on FBG observed in the PRYSMS trial. [13] A prior secondary analysis of the PRYSMS trial investigated the hypothesis that the effect of restorative yoga may be through decreased stress. However measures of both perceived stress and salivary cortisol failed to support this hypothesis. [14] MiR-363 and miR-374b have largely been studied in relation to cancer. However, miR-374b

Table. 4 Overlapping MicroRNAs By Intervention Group and Blood Glucose Outcome

\begin{tabular}{|c|c|c|c|c|c|}
\hline & & \multicolumn{2}{|c|}{ Final FBG } & \multicolumn{2}{|c|}{ Change in FBG } \\
\hline & & $\bar{C}$ & 1 & $\bar{C}$ & 1 \\
\hline \multirow[t]{5}{*}{ MicroRNAs } & let-7c & $x$ & & $x$ & \\
\hline & miR-363 & $x$ & & $x$ & \\
\hline & miR-374b & $x$ & & $x$ & \\
\hline & $\operatorname{miR}-92 a$ & $x$ & $x$ & $x$ & $x$ \\
\hline & miR-93 & $x$ & $x$ & $x$ & $x$ \\
\hline
\end{tabular}

C control group; FBG fasting blood glucose; / intervention group downregulates expression of phosphatase and tensin homologue (PTEN), which is associated with impaired insulin sensitivity, a major risk factor for T2DM. [44] An animal model study showed changes in spermatozoic let-7c levels in response to a high fat diet that were maintained in offspring and that let-7c targets mRNAs expressed in white adipose tissue. [45] The lack of association of these miRs with FBG outcomes in the intervention group may indicate that restorative yoga decreases expression of these miRs in individuals who are at risk for T2DM with potential downstream upregulation of their mRNA targets and corresponding protein products. Additional studies, including in silico analysis, of the predicted mRNA and biological pathway targets of the miRs that were associated with stretching but not yoga in individuals at risk for T2DM are needed.

\section{Conclusions}

This study focused on circulating miRs that predicted FBG at 12-months as well as change in FBG after 12months. This is one of the first studies to use a longitudinal design to assess whether miRs can predict changes in FBG over time. Rather than evaluating single miRs as predictors, we used linear modelling to identify the optimal subsets of miRs, which resulted in approximately $75 \%$ of the variance in FBG outcomes explained by the models. Among the identified miRs, several have previously been associated with risk for T2DM in other studies, and preliminary evidence suggests that others could be related to vascular endothelial complications from T2DM. Finally, we identified three miRs that were included in the set of optimal predictors for the active control group but not the intervention group. Further exploration of the function of these miRs may provide new insights about the poorly understood positive 
impact of restorative yoga on FBG. This study had limitations. Not all participants in the PRYSMS dataset had sufficient banked biospecimens available for inclusion in the current study, thus the sample may not be representative of the full PRYSMS study sample. However, as with the full PRYSMS sample, there were no significant differences in FBG at baseline between the intervention and control groups. We previously performed a discovery analysis using an independent sample of participants from the PRYSMS trial that measured the subset of reliably detectable circulating miRs in order to inform the selection of the $59 \mathrm{miR}$ included in the design of the custom assay used in the study described in this paper. Additional relevant miRs may have been excluded in the custom assay. Future studies that are powered to include a larger number of miRs may identify these additional relevant miRs as useful additions to prediction models. Future directions include replication of these findings in an independent sample to determine whether the prediction models still explain a high level of variance in FBG and functional assessment of the miRs included in the models to further understand their potential mechanistic contributions to risk for T2DM and responses to behavioral risk reduction interventions.

\section{Abbreviations}

BMI: Body mass index; FBG: Fasting blood glucose; miR: microRNA

\section{Supplementary Information}

The online version contains supplementary material available at https://doi. org/10.1186/s40364-021-00317-5.

\section{Additional file 1 \\ Additional file 2: \\ Additional file 3: \\ Additional file 4: \\ Additional file 5: \\ Additional file 6:}

\section{Acknowledgements}

Xingyue Gong contributed to descriptive statistics and prepared Fig. 1.

\section{Authors' contributions}

EF was the Principal Investigator of the grant that supported this project. EF conceived of and executed the overall study and design, oversaw all molecular data collection, directed the analysis plan, and wrote and revised the manuscript. IEA contributed to the analysis plan, performed all statistical modeling, contributed to the interpretation of the results, and approved the final manuscript. AM was the Principal Investigator of the PRYSMS trial and oversaw all clinical data collection, contributed to the design and interpretation of results for this study, and approved the final manuscript. BEA provided overall scientific guidance for this study, contributed to interpretation of the results, and approved the final manuscript. The author(s) read and approved the final manuscript.

\section{Funding}

Dr. Flowers was supported by the National Center for Advancing Translational Sciences of the National Institutes of Health grant number KL2TR000143 and the Hellman Family Foundation. The PRYSMS study was supported by the National Center for Complementary and Alternative Medicine of the National Institutes of Health grant number R01AT004569. Molecular data collection from the PRYSMS study was supported by the National Institute of Diabetes, Digestive, and Kidney Disease of the National Institutes of Health grant number R21DK117346. Dr. Kanaya is supported by National Heart Lung, and Blood Institute of the National Institutes of Health grant number 2K24HL112827.

\section{Availability of data and materials}

The datasets generated and/or analyzed during the current study are not publicly available as there are no public repositories for this type of dataset. The data are available from the corresponding author on reasonable request.

\section{Declarations}

Ethics approval and consent to participate

The parent trial and the secondary analysis reported in this manuscript were approved by the Institutional Review Board at UC San Francisco. All participants provided informed consent.

\section{Consent for publication}

N/A.

Competing interests

The authors declare that they have no competing interests.

\section{Author details}

${ }^{1}$ Department of Physiological Nursing, University of California, San Francisco, 2 Koret Way, \#605L, CA 94143-0610 San Francisco, USA. ${ }^{2}$ Institute for Human Genetics, University of California, San Francisco, 2 Koret Way, \#605L, CA 94143-0610 San Francisco, USA. ${ }^{3}$ Department of Epidemiology and Biostatistics, University of California, San Francisco, USA. ${ }^{4}$ Department of Medicine, University of California, San Francisco, USA. ${ }^{5}$ Bluestone Center for Clinical Research, New York University, New York, USA. ${ }^{6}$ Department of Oral and Maxillofacial Surgery, New York University, New York, USA.

Received: 3 May 2021 Accepted: 27 July 2021

Published online: 23 August 2021

\section{References}

1. Saeedi P, Petersohn I, Salpea P, Malanda B, Karuranga S, Unwin N, et al. Global and regional diabetes prevalence estimates for 2019 and projections for 2030 and 2045: Results from the International Diabetes Federation Diabetes Atlas, 9(th) edition. Diabetes Res Clin Pract. 2019;157:107843.

2. National Diabetes Statistics Report. Atlanta, GA: Centers for Diseaes Control and Prevention, U.S. Department of Health and Human Services; 2017.

3. Manolio TA, Collins FS, Cox NJ, Goldstein DB, Hindorff LA, Hunter DJ, et al. Finding the missing heritability of complex diseases. Nature. 2009;461(7265): $747-53$.

4. Xue A, Wu Y, Zhu Z, Zhang F, Kemper KE, Zheng Z, et al. Genome-wide association analyses identify 143 risk variants and putative regulatory mechanisms for type 2 diabetes. Nat Commun. 2018;9(1):2941.

5. 2. Classification and Diagnosis of Diabetes: $<\mathrm{em}>$ Standards of Medical Care in Diabetes—2020</em>. Diabetes Care. 2020;43(Supplement 1):S14-S31.

6. Flowers E, Won GY, Fukuoka Y. MicroRNAs associated with exercise and diet: a systematic review. Physiol Genom. 2015:47(1):1-11.

7. Parr EB, Camera DM, Burke LM, Phillips SM, Coffey VG, Hawley JA, et al. Circulating MicroRNA Responses between 'High' and 'Low' Responders to a 16-Wk Diet and Exercise Weight Loss Intervention. PLOS ONE. 2016;11: e0152545.

8. Vaishya S, Sarwade RD, Seshadri V. MicroRNA, Proteins, and Metabolites as Novel Biomarkers for Prediabetes, Diabetes, and Related Complications. Frontiers in Endocrinology. 2018;9:180.

9. Jahantigh D, Mirani Sargazi F, Sargazi S, Saravani R, Ghazaey Zidanloo S, Heidari Nia M, et al. Relationship between Functional miR-143/145 Cluster Variants and Susceptibility to Type 2 Diabetes Mellitus: A Preliminary CaseControl Study and Bioinformatics Analyses. Endocr Res. 2021:1-11.

10. Higuchi C, Nakatsuka A, Eguchi J, Teshigawara S, Kanzaki M, Katayama A, et al. Identification of circulating miR-101, miR-375 and miR-802 as biomarkers for type 2 diabetes. Metabolism. 2015;64(4):489-97. 
11. Flowers E, Aouizerat BE, Abbasi F, Lamendola C, Grove KM, Fukuoka Y, et al. Circulating microRNA-320a and microRNA-486 predict thiazolidinedione response: Moving towards precision health for diabetes prevention. Metabolism. 2015.

12. Flowers E, Gadgil M, Aouizerat BE, Kanaya AM. Circulating micrornas associated with glycemic impairment and progression in Asian Indians. Biomark Res. 2015;3(1):1-8.

13. Kanaya AM, Araneta MR, Pawlowsky SB, Barrett-Connor E, Grady D,

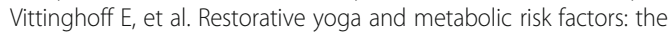
Practicing Restorative Yoga vs. Stretching for the Metabolic Syndrome (PRYS MS) randomized trial. J Diabetes Complications. 2014;28(3):406-12.

14. Corey SM, Epel E, Schembri M, Pawlowsky SB, Cole RJ, Araneta MR, et al. Effect of restorative yoga vs. stretching on diurnal cortisol dynamics and psychosocial outcomes in individuals with the metabolic syndrome: The PRYSMS randomized controlled trial. Psychoneuroendocrinology. 2014;49 C: 260-71

15. Alberti KG, Eckel RH, Grundy SM, Zimmet PZ, Cleeman Jl, Donato KA, et al. Harmonizing the metabolic syndrome: a joint interim statement of the International Diabetes Federation Task Force on Epidemiology and Prevention; National Heart, Lung, and Blood Institute; American Heart Association; World Heart Federation; International Atherosclerosis Society; and international association for the Study of Obesity. Circulation. 2009; 120(16):1640-5.

16. Friedewald WT, Levy RI, Fredrickson DS. Estimation of the concentration of low-density lipoprotein cholesterol in plasma, without use of the preparative ultracentrifuge. Clin Chem. 1972;18(6):499-502.

17. Allen M. The SAGE encyclopedia of communication research methods. Los Angeles: SAGE; 2017. 4 volumes p.

18. Vandesompele J, De Preter K, Pattyn F, Poppe B, Van Roy N, De Paepe A, et al. Accurate normalization of real-time quantitative RT-PCR data by geometric averaging of multiple internal control genes. Genome Biol. 2002; 3(7):RESEARCH0034.

19. Agarwal V, Bell GW, Nam JW, Bartel DP. Predicting effective microRNA target sites in mammalian mRNAs. Elife. 2015;4.

20. Guo L, Yang S, Zhao Y, Zhang H, Wu Q, Chen F. Global analysis of miRNA gene clusters and gene families reveals dynamic and coordinated expression. Biomed Res Int. 2014;2014:782490.

21. Chou CH, Shrestha S, Yang CD, Chang NW, Lin YL, Liao KW, et al. miRTarBase update 2018: a resource for experimentally validated microRNAtarget interactions. Nucleic Acids Res. 2018;46(D1):D296-302.

22. Kanehisa M, Goto S. KEGG: kyoto encyclopedia of genes and genomes. Nucleic Acids Res. 2000;28(1):27-30

23. Majithia AR, Flannick J, Shahinian P, Guo M, Bray MA, Fontanillas P, et al. Rare variants in PPARG with decreased activity in adipocyte differentiation are associated with increased risk of type 2 diabetes. Proc Natl Acad Sci U S A. 2014;111(36):13127-32.

24. Flowers E, Kanaya AM, Fukuoka Y, Allen IE, Cooper B, Aouizerat BE. Preliminary evidence supports circulating microRNAs as prognostic biomarkers for type 2 diabetes. Obes Sci Pract. 2017:3(4):446-52

25. Chen YH, Heneidi S, Lee JM, Layman LC, Stepp DW, Gamboa GM, et al. miRNA-93 inhibits GLUT4 and is overexpressed in adipose tissue of polycystic ovary syndrome patients and women with insulin resistance. Diabetes. 2013;62(7):2278-86.

26. Fox CS, Liu Y, White CC, Feitosa M, Smith AV, Heard-Costa N, et al. Genomewide association for abdominal subcutaneous and visceral adipose reveals a novel locus for visceral fat in women. PLoS Genet. 2012;8(5):e1002695.

27. Huang X, Liu G, Guo J, Su Z. The PI3K/AKT pathway in obesity and type 2 diabetes. Int J Biol Sci. 2018;14(11):1483-96.

28. Langi G, Szczerbinski L, Kretowski A. Meta-Analysis of Differential miRNA Expression after Bariatric Surgery. J Clin Med. 2019;8:8.

29. Esteves JV, Enguita FJ, Machado UF. MicroRNAs-Mediated Regulation of Skeletal Muscle GLUT4 Expression and Translocation in Insulin Resistance. J Diabetes Res. 2017;2017:7267910.

30. Shi C, Huang F, Gu X, Zhang M, Wen J, Wang X, et al. Adipogenic miRNA and meta-signature miRNAs involved in human adipocyte differentiation and obesity. Oncotarget. 2016;7(26):40830-45.

31. Docherty NG, Murphy M, Martin F, Brennan EP, Godson C. Targeting cellular drivers and counter-regulators of hyperglycaemia- and transforming growth factor-beta1-associated profibrotic responses in diabetic kidney disease. Exp Physiol. 2014;99(9):1154-62.
32. Essandoh K, Li Y, Huo J, Fan GC. MiRNA-Mediated Macrophage Polarization and its Potential Role in the Regulation of Inflammatory Response. Shock. 2016;46(2):122-31.

33. Sun $B$, Cao Q, Meng M, Wang X. MicroRNA-186-5p serves as a diagnostic biomarker in atherosclerosis and regulates vascular smooth muscle cell proliferation and migration. Cell Mol Biol Lett. 2020;25:27.

34. Xing X, Li Z, Yang X, Li M, Liu C, Pang Y, et al. Adipose-derived mesenchymal stem cells-derived exosome-mediated microRNA-342-5p protects endothelial cells against atherosclerosis. Aging. 2020;12(4):3880-98.

35. Gao C, Qian H, Shi Q, Zhang H. MicroRNA-363-3p serves as a diagnostic biomarker of acute myocardial infarction and regulates vascular endothelial injury by targeting KLF2. Cardiovasc Diagn Ther. 2020;10(3):421-30.

36. Wang W, Ma F, Zhang H. MicroRNA-374 is a potential diagnostic biomarker for atherosclerosis and regulates the proliferation and migration of vascular smooth muscle cells. Cardiovasc Diagn Ther. 2020;10(4):687-94.

37. Chang YJ, Li YS, Wu CC, Wang KC, Huang TC, Chen Z, et al. Extracellular MicroRNA-92a Mediates Endothelial Cell-Macrophage Communication. Arterioscler Thromb Vasc Biol. 2019;39(12):2492-504.

38. Liu X, Wang Y, Zhang X, Zhang X, Guo J, Zhou J, et al. MicroRNA-296-5p promotes healing of diabetic wound by targeting sodium-glucose transporter 2 (SGLT2). Diabetes Metab Res Rev. 2019;35(2):e3104.

39. Xiao D, Hu Y, Fu Y, Wang R, Zhang H, Li M, et al. Emodin improves glucose metabolism by targeting microRNA-20b in insulin-resistant skeletal muscle. Phytomedicine. 2019:59:152758.

40. Wang S, Du S, Lv Y, Wang W, Zhang F. Elevated microRNA-20b-3p and reduced thioredoxin-interacting protein ameliorate diabetic retinopathy progression by suppressing the NLRP3 inflammasomes. IUBMB Life. 2020; 72(7):1433-48

41. Zhen W, Hui D, Wenying S, Yulong S. MicroRNA-20b-5p regulates propofolpreconditioning-induced inhibition of autophagy in hypoxia-andreoxygenation-stimulated endothelial cells. J Biosci. 2020;45.

42. Thibonnier M, Esau C, Ghosh S, Wargent E, Stocker C. Metabolic and energetic benefits of microRNA-22 inhibition. BMJ Open Diabetes Res Care. 2020;8(1).

43. Wang $\mathrm{C}$, Liu G, Yang H, Guo S, Wang H, Dong Z, et al. MALAT1-mediated recruitment of the histone methyltransferase $\mathrm{EZH} 2$ to the microRNA-22 promoter leads to cardiomyocyte apoptosis in diabetic cardiomyopathy. Sci Total Environ. 2020:142191.

44. Mitchell F, Diabetes. PTEN mutations increase insulin sensitivity and obesity. Nat Rev Endocrinol. 2012;8(12):698

45. de Castro Barbosa T, Ingerslev LR, Alm PS, Versteyhe S, Massart J, Rasmussen $M$, et al. High-fat diet reprograms the epigenome of rat spermatozoa and transgenerationally affects metabolism of the offspring. Mol Metab. 2016; 5(3):184-97.

\section{Publisher's Note}

Springer Nature remains neutral with regard to jurisdictional claims in published maps and institutional affiliations.

Ready to submit your research? Choose BMC and benefit from:

- fast, convenient online submission

- thorough peer review by experienced researchers in your field

- rapid publication on acceptance

- support for research data, including large and complex data types

- gold Open Access which fosters wider collaboration and increased citations

- maximum visibility for your research: over $100 \mathrm{M}$ website views per year

At $\mathrm{BMC}$, research is always in progress.

Learn more biomedcentral.com/submission 